$\begin{array}{cl}\begin{array}{cl}\text { Revue } \\ \text { de Ihistoire }\end{array} & \text { Revue de l'histoire des religions } \\ \text { des religions } & 4 \mid 2010 \\ & \text { Qu'est-ce qu'un « paysage religieux »? }\end{array}$

Jardins cultuels et rites féminins d'adolescence. L'autochtonie athénienne en ses sanctuaires paysagers

Cultic Gardens and Rites of Female Adolescence: Athenian Autochthony and Landscape Architecture

\title{
Claude Calame
}

\section{OpenEdition}

Journals

\section{Édition électronique}

URL : http://journals.openedition.org/rhr/7663

DOI : $10.4000 /$ rhr.7663

ISSN : 2105-2573

\section{Éditeur}

Armand Colin

\section{Édition imprimée}

Date de publication : 1 décembre 2010

Pagination : 459-479

ISBN : 978-2200-92658-8

ISSN : 0035-1423

\section{Référence électronique}

Claude Calame, « Jardins cultuels et rites féminins d'adolescence. L'autochtonie athénienne en ses sanctuaires paysagers », Revue de l'histoire des religions [En ligne], 4 | 2010, mis en ligne le 01 décembre 2013, consulté le 20 avril 2019. URL : http://journals.openedition.org/rhr/7663; DOI : $10.4000 /$ rhr.7663 


\section{Jardins cultuels et rites féminins d'adolescence. L'autochtonie athénienne en ses sanctuaires paysagers}

Dans un bref retour sur le rituel de l'arrhéphorie et sur le récit étiologique de la courotrophie manquée des Cécropides, il s'agit d'explorer les fonctions symboliques d'une géographie cultuelle intégrant des éléments végétaux et d'expliciter les relations sémantiques complexes que ces lieux de culte, en leur configuration paysagère, entretiennent avec les divinités qui y sont vénérées, avec les pratiques rituelles qu'ils accueillent, et avec les récits fondateurs qui en justifient l'institution. Est en jeu une représentation narrative, rituelle et spatiale de l'aspect de fécondité politique de l'autochtonie revendiquée par les Athéniens.

\section{Cultic Gardens and Rites of Female Adolescence : Athenian Autochthony and Landscape Architecture}

A brief reexamination of the Athenian ritual of arrephoria and of Erichthonius' etiological narrative of the failed kourotrophy of the daughters of Cecrops leads to an exploration of the symbolic functions of a devotional geography containing elements of plant life and to a clarification of the complex semantic relationships which link worship settings, through their landscape component, to the gods venerated and the rituals performed in them, and to the foundation stories that justify such cults. At issue is a narrative, ritual and spatial representation of the political fertility of the Athenian claim for autochthony. 
On le sait, le sentiment pour le paysage en tant que tel ne semble pas attesté en Grèce antique. En revanche très nombreux sont les espaces végétaux qui accueillent une divinité, dans le récit héroïque et dans la pratique cultuelle, dans le «mythe » et dans le «rite». Selon les valeurs culturelles attribuées aux végétaux qui les composent, ces configurations paysagères de légende ou de culte partagent avec la divinité qu'elles abritent des qualités spécifiques ${ }^{1}$. Par référence très limitée à deux études précédentes, rappelons ici en guise d'introduction les affinités qui associent, du point de vue paysager, les prairies fleuries évoquant les pouvoirs séducteurs d'Aphrodite et les prés élyséens qu'au-delà de l'Hadès protège Perséphone. D'une part le sanctuaire-jardin dans lequel des vers composés par Sappho invitent Aphrodite à intervenir pour partager avec ses jeunes servantes nectar et réjouissances musicales; de l'autre les prairies et les bois sacrés où Perséphone accueille les âmes qui sont porteuses des textes rituels et poétiques transcrits sur des lamelles funéraires d'or, véritables passeports pour l'au-delà2.

Si la prairie de Perséphone relève d'une représentation poétique que l'on retrouve par exemple chez Pindare, le sanctuaire paysager décrit par Sappho correspond certainement à un lieu de culte réel. Dans les deux cas, c'est la récitation ou le chant des vers concernés qui permet d'accomplir le passage indiqué; dans les deux cas il s'agit de poèmes de culte dont la performance contribue à l'accomplissement rituel du passage spatial et au changement de statut - initiation cultuelle aux plaisirs d'Aphrodite pour la poésie de Sappho, accès rituel à une forme d'immortalité pour les bienheureux initiés méritant la compagnie de Perséphone pour l'éternité.

1. Sur les représentations grecques de ce qui est pour nous le paysage, voir la contribution de Ada Cohen, « Mythic Landscapes of Greece »; les usages du paysage dans les mythes et les cultes grecs sont au centre du bon chapitre de Richard Buxton, Imaginary Greece, p. 80-113. - La présente étude a aussi fait l'objet d'une communication à la rencontre internationale "Geschichte und Gegenwart von Bau- und Gartenkultur » organisée par la Fakultät für Architektur und Landschaft de l'Université Leibniz de Hanovre (4-6 octobre 2007); elle s'inscrit dans une recherche plus vaste sur les cultes athéniens impliquant jeunes filles et femmes.

2. Sappho, fr. 2 Voigt et lamelle de Thurii A 4 Zuntz $=$ Orphica fr. 487 Bernabé; $c f$. Claude Calame, Pratiques poétiques de la mémoire, p. 263-271, et « Jardins d'amour et prairies de l'au-delà », avec les références bibliographiques de rigueur sur deux textes à l'interprétation controversée. 
Appelant la présence d'Aphrodite dans le sanctuaire-jardin qui lui est consacré, le poème de Sappho nous invite donc à partager avec la déesse des espaces paysagers bien réels, des espaces construits, des espaces de culte, des espaces religieux où s'accomplit un passage rituel. Ce sont des espaces d'architecture essentiellement végétale qui accueillent une action cultuelle liée à la transition d'un état à un autre, à ce qu'on appelle en anthropologie un « rite de passage »; dans le régime polythéiste propre à chaque cité grecque, le changement rituel de statut, la transformation identitaire impliquent l'intervention, dans chaque cas, d'une divinité spécifique, parfois accompagnée d'un héros ${ }^{3}$. Par l'intermédiaire des récits qui expliquent l'origine du rituel et par métaphores interposées, nombreuses sont les qualités partagées entre la divinité agente du passage avec ses qualités et modes d'action propres, les acteurs du récit étiologique qui fonde la pratique rituelle, le statut des protagonistes de la transition et le contexte d'architecture végétale qui accueille cet acte cultuel, impliquant l'actualisation des valeurs culturelles attribuées aux végétaux concernés. De ce point de vue, l'espace cultuel athénien est particulièrement riche de sanctuaires paysagers; ils sont les lieux de différents types de rites de passage destinés en particulier aux jeunes filles. L'organisation de ces sanctuaires nous confronte à une "landscape architecture » concrète servant de support à différents processus d' « anthropopoiésis », de fabrication culturelle et sociale (par la mémoire narrative et poétique ainsi que par la pratique rituelle) des êtres humains, hommes et femmes, avec leur identité spécifique $^{4}$. Ces processus anthropopoiétiques opèrent en particulier à travers les valeurs métaphoriques attribuées aux éléments du pay-

3. Les différentes formes assumées par les rites de passage dans les cités grecques, en particulier en ce qui concerne les pratiques de l'initiation tribale réservée aux adolescents ou aux adolescentes, sont désormais soumises aux études critiques réunies dans Mark W. Padilla (ed.), Rites of Passage in Ancient Greece (notamment celle que j'ai reprise et développée en français dans Francis Affergan et alii, Figures de l'humain, p. 127-173) ainsi que dans David Dodd \& Christopher Faraone (ed.), Initiation in Ancient Greek Rituals.

4. Le concept opératoire d' "anthropopoiésis » a été développé notamment par Francesco Remotti dans Francis Affergan et alii, Figures de l'humain, p. 17-74 et 279-306; sur les bases de l'analyse des fonctions, modes d'action et champs d'intervention des divinités en régime polythéiste, voir les références bibliographiques données dans Claude Calame, «L'histoire comparée des religions et la construction d'objets différenciés », p. 216-225. 
sage architectural et cultuel; ils définissent et rendent actives, par l'exemple héroïque et par la pratique rituelle, des identités politiques de l'ordre du « gender».

\section{LE RITUEL DE L'ARRHÉPHORIE ET SON ÉTIOLOGIE}

À commencer par Aphrodite, et par Aphrodite aux Jardins dont le sanctuaire a été identifié grâce à une dédicace à Éros sur une terrasse située dans le flanc de la falaise septentrionale de l'Acropole $^{5}$. À l'occasion de sa visite du roc sacré, Pausanias met ce sanctuaire en relation avec la présence de l'olivier sur l'Acropole, dans l'enclos aménagé devant le temple d'Athéna Polias qui est devenu pour nous l'Érechthéion; c'est l'olivier que la déesse avait fait pousser sur l'Acropole dans sa rivalité avec Poséidon pour la possession de l'Attique. Puis, dans l'immédiat voisinage de l'arbre toujours vivace et à l'intérieur de cette enceinte sacrée, le Périégète signale le petit sanctuaire consacré à Pandrosos; cette héroïne athénienne est la seule des trois filles de Cécrops à ne pas s'être rendue coupable envers Athéna de la faute que nous verrons.

Or, contrairement à son habitude, Pausanias rapporte à ce propos non pas un récit héroïque, mais les gestes d'une action rituelle. Le rite est accompli par deux des quatre arrhéphores, les très jeunes filles auxquelles était confié le tissage du péplos destiné à Athéna Polias à l'occasion des Panathénées. Peu avant la célébration de cette grande fête civique, les deux filles quittaient de nuit leur demeure située sur l'Acropole; elles portaient sur la tête les objets secrets que leur avait confiés la prêtresse d'Athéna. En empruntant un passage souterrain, elles déposaient ces objets dans le sanctuaire d'Aphrodite " dans les Jardins », adossé au flanc septentrional du rocher de l'Acropole; elles en revenaient en apportant un autre objet, lui aussi soigneusement dérobé à leur regard. À la suite de ce double parcours rituel, les deux arrhéphores concernées laissaient définitivement la maison édifiée à leur intention pour être remplacées par deux autres jeunes filles ${ }^{6}$.

5. On trouvera chez Vinciane Pirenne-Delforge, L'Aphrodite grecque, p. 48-62, les quelques rares informations que nous avons sur le sanctuaire et le culte dédiés à Aphrodite aux Jardins. De manière générale, sur les cultes jardiniers rendus à Aphrodite voir André Motte, Prairies et Jardins, p. 121-137.

6. Pausanias, 1, 27, 2-3; pour la bibliographie au sujet de ce rituel, cf. infra notes 12 et 13 . 
Cet étrange rituel peut être mis en relation étiologique avec l'un des récits hérö̈ques qui expliquent la délimitation du territoire de l'Attique et la fondation d'Athènes tout en légitimant les prétentions des Athéniens à l'autochtonie. La légende athénienne raconte en effet la naissance du petit Érichthonios, surgi des entrailles du sol de l'Attique après que le sperme d'Héphaïstos, dans sa vaine poursuite amoureuse de la vierge Athéna, eut fécondé Terre; après avoir fait de Terre la mère biologique d'Érichthonios et d'Athéna sa mère symbolique, le récit se poursuit avec la courotrophie manquée de l'enfant par deux des trois filles du premier roi autochtone de l'Attique, Cécrops. Cet épisode sert sans doute d'explication, d'ordre étiologique, pour l'étrange pratique rituelle rapportée par Pausanias.

Avant d'y revenir dans le détail, remarquons pour l'instant que, par l'intervention de la fécondité d'une terre qui apparaît également comme la mère des futurs citoyens autochtones, ce récit s'inscrit dans la saga de la rivalité d'Athéna et de Poséidon pour la délimitation, la définition et le contrôle du territoire de l'Attique. La légende contribue à l'institution et à la définition du pouvoir partagé par les deux futurs dieux tutélaires de la cité d'Athènes tel qu'il était figuré sur le fronton occidental du Parthénon de l'époque classique : d'un coup de son trident, Poséidon faisait surgir du roc de l'Acropole la source d'eau salée dénommée plus tard Mer Éréchthéide et insérée, comme trace de la mémoire civique, dans le futur Érechthéion; Athéna quant à elle y faisait croître le premier olivier qui sera rituellement inclus dans le périmètre cultuel où, auprès du même Érechthéion, s'élèvera le petit sanctuaire héroïque réservé à la Cécropide Pandrosos (et mentionné par Pausanias) ${ }^{7}$.

Ce qui est en jeu ici, ce sont bien les capacités productrices du sol de l'Attique, roc de l'Acropole inclus ! Ces virtualités sont illustrées par les gestes symboliques et merveilleux accomplis dans la Cité haute d'Athènes même; elles sont inscrites dans un paysage cultuel encore à visiter: olivier d'Athéna et mer Érechthéide surgissant tous deux du compact et aride rocher de la sainte Acropole. Rete-

7. Le récit est évoqué par Hérodote, 8,55 et par Thucydide, $2,15,1, c f$. aussi Pseudo-Apollodore, Bibliothèque, 3, 14, 1 (sur Erekhtheìs thálassa); pour la scène représentée sur le fronton occidental du Parthénon et sur les différentes versions d'une rivalité fondatrice, voir Robert Parker, "Myths of Early Athens ", p. 198-199, et J. M. Hurwit, The Athenian Acropolis, p. 174-178 et 292-293. 
nons pour le moment que le rituel décrit par Pausanias fait passer les deux arrhéphores par un couloir dont l'obscurité contraste avec les couleurs du sanctuaire-jardin consacré à Aphrodite; par ce couloir sombre, les deux jeunes arrhéphores pénètrent dans les entrailles de ce roc que les interventions d'Athéna et de Poséidon ont rendu symboliquement fertile, à l'exemple de la terre de l'Attique.

\section{RÉCITS HÉROÏQUES : LES ESPACES DE LA COUROTROPHIE D'ÉRICHTHONIOS}

La dispute entre Athéna et Poséidon nous invite donc à retourner à la figure d'Érichthonios. Engendré par le sperme d'Héphaïstos tombé sur le sol de l'Attique dans sa poursuite amoureuse de la vierge Athéna, le petit enfant a donc pour mère symbolique la déesse tutélaire de la cité et pour mère biologique Gé, incarnation de son territoire. Né des entrailles de la terre, l'enfant deviendra le roi de l'Attique sous le nom d'Érechthée. À son règne, la tradition attache la fondation des Mystères d'Éleusis avant que le héros royal ne retrouve les profondeurs du sol dont il est né. À l'occasion de la guerre menée à Éleusis contre le Thrace Eumolpe, le fils de Poséidon, Érechthée sera en effet frappé par le trident du dieu qui ébranle les entrailles de la terre et les profondeurs de la mer, puis enfoui dans la terre de l'Attique. Par la volonté de l'Athéna mise en scène dans la tragédie qu'Euripide consacre à cet épisode fondateur de l'autochtonie athénienne, Érechthée sera héroïsé comme "parèdre " de Poséidon et vénéré en tant que tel dans le futur Érechthéion. À la naissance autochtone succède une mort hérö̈que, elle aussi autochtone ; toutes deux s'inscrivent dans le contexte général de la lutte légendaire entre Poséidon et Athéna pour le contrôle de la terre de l'Attique avec ses habitantes et pour la définition de ses qualités propres ${ }^{8}$.

8. Euripide, Érechthée, fr. 370, 71-74 Kannicht; sur la naissance d'Érichthonios et sur les différentes versions de la guerre légendaire conduite contre Érechthée par Eumolpe, le roi de Thrace ou le souverain d'Éleusis, voir Robert Parker, "Myths of Early Athens », p. 200-204; sur le sens de sa mort « autochtone », $c f$. Sonia Darthou, « Retour à la terre », p. 79-83. Le nom de culte Poséidon-Érechthée est attesté dans l'inscription $I G \mathrm{I}^{2}, 580=\mathrm{IG} \mathrm{I}^{3} .2,873$ (qui date du milieu du $v^{\mathrm{e}}$ siècle); les martúria de l'histoire fondatrice de l'Attique et d'Athènes qui sont vénérés dans le temple d'Athéna Polias dénommé Érechthéion 
Or c'est à Pausanias lui-même que nous devons, sous forme mythographique, le détail du récit de la courotrophie d'Érichtonios : «On affirme qu'Athéna confia Érichthonios à Aglauros et à ses sœurs Hersé et Pandrosos; la déesse l'avait placé dans un coffre en leur interdisant de s'intéresser à son contenu; on raconte que Pandrosos obéit, mais que les deux autres ouvrirent le coffre, qu'à la vue d'Érichthonios elle furent saisies de folie et qu'elles se jetèrent du haut de la falaise la plus raide de l'Acropole. » Tel est le récit que rapporte le Périégète pour rendre compte, dans une perspective étiologique, de la fondation du témenos réservé à Aglauros. Quant au sanctuaire consacré à sa sœur Pandrosos, Pausanias le mentionnera plus avant dans sa visite, pour nous raconter le rituel de l'arrhéphorie, dans l'étrange description que nous avons déjà rapportée ${ }^{9}$.

En deçà de ce résumé mythographique tardif, la tragédie attique est là pour donner des couleurs, une pragmatique et une conjoncture historique et culturelle au récit héroïque. Par bribes, l'histoire de la courotrophie d'Érichthonios nous est rappelée dans la tragédie athénienne, hautement politique, que pendant la guerre du Péloponnèse Euripide consacra à Ion, le fils naturel d'Apollon et de la dernière des filles d'Érechthée. Dans le prologue du drame, le dieu de Delphes nous rappelle comment l'enfant a été exposé par sa mère, jeune fille; à propos de cette exposition en un lieu qui correspond à la falaise nord-orientale de l'Acropole et qui est dénommé " les Grandes Roches », le dieu évoque la naissance du grand-père, Érichthonios «né de la terre » (gegenés). À propos de cette naissance autochtone, le dieu de Delphes rappelle que « la fille de Zeus avait placé auprès du nouveau-né deux serpents en tant que gardes du corps invitant les Aglaurides, des jeunes filles, à en prendre soin ». Dans une perspective étiologique, Apollon précise de plus que sur ce récit se fonde la coutume propre aux

sont mentionnés en particulier par Pausanias (cf. 1, 26, 5 et 27, 1-2) à l'occasion de sa visite de l'Acropole.

9. Pausanias, 1, 18, 2; voir aussi Pseudo-Apollodore, Bibliothèque 3, 14, 6; autres versions de la légende chez Walter Burkert, «Kekropidensage und Arrhephoria », p. 10-12. Sur le sanctuaire consacré à Aglauros, cf. infra note 30. Le riche dossier iconographique, centré sur la naissance chthonienne d'Érichthonios et qui montre en général Athéna casquée, est déployé et commenté par Pierre Brulé, La Fille d'Athènes, p. 68-79; voir aussi Uta Kron, « Aglauros, Herse, Pandrosos », p. 287-290 ainsi que H. Alan Shapiro, « The Daughters of Cecrops ». 
«descendants d'Érechthée », c'est-à-dire aux Athéniens, d'élever les enfants au milieu de serpents d'or ${ }^{10}$. Dans un premier échange entre Ion et sa mère Créuse (qui identifie sa «terre-patrie » avec la cité d'Athènes), on apprend encore qu'Érichthonios a été accueilli au moment de sa naissance de la terre par Athéna elle-même, la fille de Zeus; la déesse recueillit le bébé dans ses bras de vierge, « elle qui ne l'avait pas engendré ». Puis la déesse confia le nouveau-né, dérobé à la vue, aux filles de Cécrops et d'Aglauros (la mère porte le même nom que l'une de ses filles); elles ne purent s'empêcher d'ouvrir la corbeille de la déesse et elles payèrent leur désobéissance d'un accès de folie au cours duquel elles se précipitèrent du haut de l'Acropole, dans la falaise dite « des Grandes Roches ». Il appartiendra enfin au groupe choral des servantes (athéniennes) d'évoquer encore une fois ces Grandes Roches. En leur sommet, non loin de la grotte de Pan où le petit Ion fut exposé, face aux temples consacrés à Athéna Pallas, elles situent la danse chorale des filles de Cécrops et d'Aglauros; au son de la syrinx, ces jeunes filles foulaient de leurs pas rythmés une étendue de gazon qui évoque toutes les prairies de séduction accueillant tour à tour les amours d'hérö̈nes de légende et les pratiques rituelles réservées aux jeunes filles nubiles ${ }^{11}$. Nous voici donc de retour auprès d'un sanctuaire au fort caractère paysager : grotte et aire de danse chorale couverte d'une herbe tendre.

\section{L'ARRHÉPHORIE EN RITE D'INITIATION TRIBALE?}

Assorti de son récit étiologique sur la courotrophie manquée d'Érichthonios, le rituel de l'arrhéphorie, on se l'imagine, a déjà fait l'objet de commentaires nombreux, anciens et modernes ${ }^{12}$. Pour faire bref, rappelons que, dans la perspective de l'anthropologie

10. Euripide, Ion 21-26; quant à la topographie de la falaise nord-orientale de l'Acropole dénommée « Grandes Roches », cf. encore infra note 30.

11. Euripide, Ion, 260-284 et 492-509; les « temples de Pallas » correspondent sans doute, en cette fin du v $v^{\mathrm{e}}$ siècle, au Parthénon et à l'Érechthéion. La situation topographique de la Grotte de Pan est précisée par J. M. Hurwit, The Athenian Acropolis, p. 38 avec fig. 7; pour les prairies de la séduction, voir Claude Calame, L'Éros dans la Grèce antique, p. 173-197.

12. Les différentes interprétations du rituel décrit par Pausanias, 1, 27, 3 sont résumées par Pierre Brulé, La Fille d'Athènes, p. 83-90; voir aussi Claude Calame, L'Éros dans la Grèce antique, p. 193-197. 
contemporaine de la religion grecque, on s'est volontiers appuyé sur l'insertion du service de l'arrhéphorie dans la séquence des quatre moments rituels marquant la carrière adolescente de l'Athénienne idéale pour donner de ce parcours une interprétation initiatique. Ce parcours anthropopoiétique en quatre étapes est évoqué par les choreutes de la Lysistrata d'Aristophane pour donner de ce parcours rituel une interprétation initiatique : dès l'âge de sept ans arrhéphore, à dix broyeuse de grain pour l'Archégète, ensuite ourse portant la robe de safran aux Brauronies, enfin canéphore avec le statut de la « belle jeune fille»-qui signifie pour la jeune femme l'accession à la maturité érotique de la puberté achevée ${ }^{13}$. Ainsi, du côté de la pratique rituelle, les objets secrets transportés par les arrhéphores, le passage par un couloir sombre et sauvage, la transition d'un lieu de culte consacré à une déesse vierge vers un sanctuaire consacré à la divinité de la sexualité adulte semblent indiquer un itinéraire d'ordre initiatique, avec ses trois phases canoniques de séparation, de marge et d'agrégation. Du côté de la légende héroïque, la découverte prématurée d'un nouveau-né, l'accès de folie infligé par la divinité à des jeunes filles et leur mort par précipitation évoquent aussi les morts adolescentes que mettent en scène les récits mis en relation avec les rites de passage réservés aux adolescents et adolescentes. Tout dans le rituel comme dans le récit semble donc inviter l'anthropologue historien de la religion grecque à placer l'arrhéphorie athénienne sous l'étiquette du rite de passage et, plus précisément, sous celle du rite d'initiation tribale ${ }^{14}$.

Néanmoins plusieurs informations, entre récit étiologique et gestes rituels, devraient prévenir l'herméneute anthropologue d'insérer le service de l'arrhéphorie parmi les rites qui marquent le passage collectif des adolescents ou des adolescentes intégrées à une classe d'âge au statut d'adulte (en tant que membres de plein droit de la communauté). À ce titre on relèvera les éléments suivants : le très jeune âge rituel des arrhéphores qui se situe entre 7 et 11 ans,

13. Aristophane, Lysistrata, 636-647; sur ce passage au texte quelque peu incertain et à l'interprétation controversée, voir en dernier lieu les remarques décisives de Franca Perusino, "Le orse di Brauron »; sur les différents statuts évoqués dans ce texte, voir les excellentes remarques de Robert Parker, Polytheism and Society, p. 218-228.

14. Voir déjà Walter Burkert, «Kekropidensage und Arrhephoria », p. 12-16 (« Mädchenweihe »); $c f$. aussi Pierre Brulé, La Fille d'Athènes, p. 138-139. 
le nombre restreint des filles prépubères et de bonne famille qui sont choisies par l'archonte-roi pour ce service religieux, le retour des jeunes filles sur l'Acropole avant de quitter définitivement le service d'Athéna; s'y ajoute, dans le récit héroïque, la mort des Cécropides qui fixe le statut des deux jeunes filles désobéissantes au moment d'une adolescence encore immature. En revanche la courotrophie narrative du nouveau-né Érichthonios, qui est destiné à devenir en tant qu'Érechthée l'un des rois légendaires et fondateurs les plus marquants de l'Attique, et la participation rituelle des arrhéphores à la préparation de la fête nationale en faveur de la déesse maîtresse de la cité engagent à une interprétation d'ordre politique; une telle lecture est encouragée par le rôle de réciprocité métaphorique assumé par la sexualité humaine d'une part et par le processus de croissance de la végétation de l'autre, dans les deux faces, narrative et rituelle, de la manifestation symbolique.

\section{LES VALEURS SYMBOLIQUES DE LA FÉCONDITÉ AUTOCHTONE}

C'est l'interprétation indigène qui nous invite dans une telle direction. Avec sa triple orthographe (arrhe-/errhe-/(h)ersephoría) la dénomination du rituel a été mise en relation étymologisante, par les Anciens, d'une part avec le caractère indicible et mystérieux des objets transportés, d'autre part avec le nom de la fille de Cécrops, Hersé, qui correspond au nom de la rosée ${ }^{15}$. Par ailleurs, un commentateur tardif indique que le rite auquel il donne le nom d'Arrhétophories a pour but de favoriser aussi bien la production des fruits que la procréation des êtres humains : il précise qu'à cette occasion, « on rapporte à la surface des objets sacrés et indicibles (árréta) faits de suif et de farine de blé, imitant des serpents et des

15. Ces différentes explications étymologisantes figurent dans différentes notices dont la référence est indiquée par Pierre Brulé, La Fille d'Athènes, p. 79-83 (également sur l'âge des filles), avec en particulier le témoignage de la scholie à Aristophane, Lysistrata, 641 (II. 4, p. 641 Holwerda) qui remonte à l'Atthidographe Istros, FGrHist. $334 \mathrm{~F} 27$; voir aussi, à propos des données lexicographiques, Guy Donnay, «L'Arrhéphorie », p. 178-186. Sur le nom de Hersé à laquelle les inscriptions substituent la figure de Courotrophos ( $c f$. infra note 30), voir Pierre Brulé, La Fille d'Athènes, p. 30-43. 
organes masculins ${ }^{16} »$. Serait-ce à dire que, plutôt qu'à un rituel d'initiation tribale, l'Arrhéphorie correspond à l'un de ces « festivals de fertilité » chers aux historiens des religions du XIX ${ }^{\mathrm{e}}$ siècle? Sans doute, par figures héroïques et métaphores interposées, l'analogie entre la productivité de la terre et l'engendrement des humains est-elle inscrite dans la séquence athénienne des « mythes d'autochtonie », mais de manière réciproque : la terre productrice parce qu'elle a été fertilisée et cultivée est comparée à la femme qui engendre et qui élève, dans un va-et-vient métaphorique déjà évoqué et que l'on aura encore à préciser ${ }^{17}$ !

Mais dans le contexte de la représentation mythique et rituelle de l'autochtonie athénienne en relation avec les filles de Cécrops, il convient d'abord de tenir compte de la nature double d'un père premier roi de l'Attique: l'ophidien Cécrops «qui déroule ses replis auprès de ses filles ", comme nous le rappelle Euripide dans l'Ion, toujours en cette même fin du $v^{\mathrm{e}}$ siècle athénien. Dans cette conjoncture culturelle le serpent est appréhendé en tant que « fils de la terre » (civique) comme le confirme l'histoire de la prise de Sardes telle que la rapporte Hérodote; la destruction de la ville de Crésus est annoncée par une apparition de serpents rapidement mangés par les chevaux qui paissaient dans les faubourgs; consultés, des devins de Carie voient dans les serpents, fils de la terre, les habitants de Sardes et dans les chevaux, animaux belliqueux venus du dehors, les soldats de Cyrus. Par naissance de la terre interposée le serpent peut donc devenir l'homologue du citoyen autochtone. Cécrops, le premier roi autochtone de l'Attique, arbore une queue de serpent; le petit Érichthonios né de l'union différée d'Athéna et d'Héphaïstos par le biais de cette même terre a pour premiers gardes du corps deux serpents; son petit-fils Ion, déposé dans une

16. Scholie à Lucien, Dialogue des courtisanes (p. 276, 13-17 Rabe); par trop centrée sur le rite de fertilité, la comparaison entre les gestes rituels des Arrhéphories, des Scirophories et des Thesmophories athéniennes est conduite par Ludwig Deubner, Attische Feste, p. 13-15 et 40-44, sur fond de rite de fertilité (voir les remarques critiques de Walter Burkert, « Kekropidensage und Arrhephoria », p. 5-9); on se référera aussi à Robert Parker, Polytheism and Society, p. 173-177 et 221-224.

17. Cf. Platon, Ménéxène, $237 \mathrm{~d}-238 \mathrm{a}$ : « car ce n'est pas terre qui a imité la femme dans la grossesse et l'enfantement, mais la femme la terre »; le texte de Platon est interprété par Nicole Loraux, Né de la terre, p. 132-135, dans une lecture littérale que corrige opportunément Stella Georgoudi, « Gaia/Gé », p. 131-133. 
corbeille analogue, porte sans doute les serpents d'or qui désormais identifient les Athéniens, "fils d'Érechthée »; et sur un cratère à colonnettes à figures rouges représentant Athéna chassant Aglauros et Hersé alors que Pandrosos se tient à l'écart dans un geste de retenue pudique, non seulement la déesse porte une égide bordée de têtes de serpent, mais l'une des deux jeunes filles tient elle-même à la main le serpent d'Érichthonios ${ }^{18}$.

Aux affinités du serpent avec la naissance des entrailles de la terre il faut ajouter la valeur fertilisante que les Grecs attribuaient, à travers différents récits, à l'eau tombant du ciel pour pénétrer le sol : humidité fécondante, sous différentes formes parmi lesquelles la pluie de Zeus ou le sperme d'Héphaïstos. La réciprocité métaphorique entre le végétal et l'humain est à nouveau entière : pluie d'or de Zeus qui métaphoriquement féconde Danaé pour donner naissance à Persée, semence d'Héphaïstos qui fertilise littéralement la terre attique d'où sortira Érichthonios; en poète savant, Callimaque qualifie l'enfant de « rosée d'Héphaïstos ${ }^{19}$ »! Cette fertilité du sol par l'élément humide qui le nourrit, deux des Cécropides en tout cas la portent indirectement dans leur nom propre : Hersé, Goutte d'eau, pour cette rosée qui est boisson et aliment pour les cigales faisant résonner l'été de leurs chants ou qui dans sa fraîcheur fait croître les arbustes de même que la poésie d'éloge grandit la valeur ${ }^{20}$; et Pandrosos, Toute Rosée, pour cette même rosée à laquelle on compare la grâce éclatante des Charites ou le tendre duvet qui, avec la croissance adolescente, couvre la joue des jeunes gens ou leurs parties ${ }^{21} \ldots$ Si eérse nous renvoie au vaste champ métaphorique de la croissance et de la floraison végétales grâce à l'écoulement d'une eau fraîche et à l'irrigation, drósos nous invite

18. Cf. Euripide, Ion, 1163-1164, puis Hérodote, 1, 78, 3 ; à propos des valeurs « chthoniennes » attribuées au serpent en Grèce classique, voir Laurent Gourmelen, Kékrops, p. 38-48, 329-349 et 393-400.

19. Selon le récit repris par exemple par Simonide, fr. 543 Page et par Pindare, Pythique, 12, 15-17; cf. Callimaque, fr. 260, 19 Pfeiffer. Cratère à colonnettes San Antonio Museum of Art Inv. Nr. 86-134G (63) : voir Alan H. Shapiro, « The Daughters of Cecrops », p. 43.

20. Pseudo-Hésiode, Bouclier, 393-396; Pindare, Némeénne, 8, 40-41; autres références et explications étymologiques chez Deborah Boedeker, Descent from Heaven, p. 52-79.

21. Pindare, Isthmique, 6, 62-64 et Aristophane, Nuées, 977-978; cf. aussi Anacreontica, 17, 9-11; autres exemples, notamment relatifs à l'inspiration poétique, chez René Nünlist, Poetologische Bildersprache, p. 186-196. 
plutôt dans celui de la délicatesse lumineuse des végétaux couverts de rosée.

Quant à Aglauros, Brillance, elle porte souvent, par métathèse, le nom de sa mère Agraulos, la Champêtre, l'épouse de Cécrops; comme la Charite Aglaia, la resplendissante Cécropide affiche dans son nom la beauté éclatante à laquelle renvoie le qualificatif aglaós, en combinaison étymologique possible avec un mot qui renverrait à l'eau courante et qui l'associerait ainsi à chacun des noms de ses deux sœurs ${ }^{22}$. D'Aglauros on sait qu'elle était la première nommée au terme du serment que les éphèbes prêtaient à l'âge de dix-huit ans dans le sanctuaire même qui lui était consacré au pied nordoriental de l'Acropole; Aglauros/Agraulos y est prise à témoin non seulement avec Hestia, Athéna, Zeus et différentes divinités de la guerre, mais aussi avec Thallô et Auxô, incarnations divines de la floraison et de la croissance, avec Hégémoné, l'une des Charites, et avec les blés, les orges, les vignes, les oliviers et les figuiers ${ }^{23}$. Dans le texte de ce serment datant $d u \mathrm{IV}^{\mathrm{e}}$ siècle, les jeunes recrues, futurs citoyens d'Athènes, sont étroitement associés aux puissances de la fertilité du sol dont ils sont nés et de la productivité agricole du territoire politique dont ils sont appelés à défendre les frontières. Processus d'anthropopoiésis rituelle pour les filles, mais également pour les jeunes gens. Dans la composition de ce paysage mythico-rituel, les végétaux soumis à la culture jouent un rôle déterminant.

\section{ESPACES CULTUELS ET COHÉRENCE PAYSAGÈRE}

$\mathrm{Du}$ point de vue cultuel, Pandrosos disposait donc face à l'Érechthéion du petit sanctuaire héroïque qu'on a déjà mentionné et qui était inclus dans l'enclos où croissait, adossé à l'Érechthéion,

22. On verra à propos de l'incertitude morphologique régnant sur le nom d'Aglauros/Agraulos et sur les spéculations étymologisantes qu'elle a suscitées l'analyse détaillée proposée par Pierre Brulé, La Fille d'Athènes, p. 28-31. On notera que dans les manuscrits de l'Ion d'Euripide orthographient en général le nom de l'épouse de Cécrops comme Agraulos (cf. vers 23 et 496).

23. Inscription d'Acharnes, p. 204 Tod; voir aussi la liste de divinités donnée par Pollux 8, 105 ; cf. Chrysis Pélékidis, Histoire de l'éphébie attique, p. 110-113, ainsi que Peter Siewert, «The Ephebic Oath », p. 109-111, qui relève les traits linguistiques et les éléments de culte qui pourraient faire remonter ce texte à l'époque pré-classique. 
l'olivier sacré. Rappelons que cet olivier miraculeux évoque, dans la lutte pour la possession divine de l'Attique, le geste de plantation arboricole d'Athéna sur le roc infertile de l'Acropole; il constitue la réponse donnée par la déesse poliade au coup du trident de Poséidon et au jaillissement non moins miraculeux de la source d'eau marine dont les traces étaient vénérées dans l'Érechthéion lui-même. Pandrosos, Toute Rosée, la jeune fille obéissante dont on peut supposer qu'elle a assumé la courotrophie d'Érichthonios, est donc la voisine cultuelle directe d'Athéna Polias ${ }^{24}$. Dans ce voisinage courotrophe, la déesse est donc représentée par l'olivier éternellement vert et brillant, l'« olivier de la cité »; cet arbre sacré renvoie, dans son constant renouvellement, à la part prise par la déesse non seulement à la productivité alimentaire de l'Attique, mais aussi à l'éducation guerrière des jeunes pousses que sont les éphèbes ${ }^{25}$ : métaphore de l'anthropopoiésis masculine dans la construction rituelle et symbolique de l'identité du citoyen-soldat. D'autre part, par l'intermédiaire du récit étiologique, serpent et rosée associent les très jeunes arrhéphores et leurs gestes rituels à l'engendrement et à la naissance (autochtone!) des futurs citoyens athéniens ${ }^{26}$. Un tel engendrement n'est possible, dans l'union sexuée, que par l'intervention d'Éros, incarnation d'un désir amoureux qui se manifeste cultuellement dans les jardins réservés à Aphrodite ${ }^{27}$.

L'ensemble topographique et métaphorique que forment les différents sanctuaires, les gestes rituels et les récits associés aux filles de Cécrops, le premier souverain encore mi-animal de l'Attique, est d'une remarquable cohérence : les éléments végétaux sont combinés avec les constructions architecturales pour inscrire dans ce

24. Cf. J. M. Hurwit, The Athenian Acropolis, p. 204-205, et Robert Parker, Polytheism and Society, p. 51-52 et 219-222. Sur les témoignages qui, par le jeu des épiclèses, associent non seulement Pandrosos mais aussi Aglauros à Athéna, cf. Emily Kearns, The heroes of Attica, p. 23-27 (avec note 81).

25. Lire en particulier Sophocle, Edipe à Colone, 701-706, pour une « configuration politico-religieuse » reconstruite avec abondance et acuité par Marcel Detienne, L'écriture d'Orphée, p. 71-84.

26. Cf. Claude Calame, L'Eros dans la Grèce antique, p. 193-197. Plutôt que de « initiation into womanhood», Emily Kearns, The Heroes of Attica, p. 24-26, pense à un rite centré sur " childbirth and childcare ».

27. Quant au rôle joué par Aphrodite et Éros dans l'accomplissement du service arrhéphorique, voir successivement Claude Bérard, Anodoi, p. 117-125, Vinciane Pirenne-Delforge, L'Aphrodite grecque, p. 48-59, et Claude Calame, L'Éros dans la Grèce antique, p. 195-197; de manière plutôt floue, Guy Donnay, «L'Arrhéphorie », p. 200-202, penche pour une « initiation à la sexualité ». 
paysage cultuel les valeurs métaphoriques de la génération et de la croissance des citoyens attachées à la fertilité du sol de l'Attique (transposée par le «mythe » sur le roc de l'Acropole!). En relation avec les récits héroïques le complexe rituel s'appuie sur une géographie cultuelle qui reprend les valeurs symboliques inscrites dans les récits étiologiques. À commencer par le sanctuaire consacré à l'obéissante Cécropide Pandrosos et adossé à l'Érechthéion auprès de l'olivier sacré, planté dans le roc de l'Acropole par Athéna. Ainsi spatialement inclus dans le périmètre de l'Érechthéion, le Pandroséion était situé au-dessus du sanctuaire réservé sur le flanc septentrional de l'Acropole à Aphrodite aux Jardins et à son assistant horticole Éros; évoquant les arrhéta rituels portés à la dérobée par les deux petites arrhéphores, les nombreux phallus de pierre retrouvés dans la zone semblent confirmer la relation directe de ce sanctuaire avec la sexualité humaine la plus explicite ${ }^{28}$.

Par ailleurs, l'enclos incluant avec l'olivier d'Athéna le petit sanctuaire consacré devant l'Érechthéion à la vierge Pandrosos se trouve à moins de trente mètres de l'édifice identifié comme la maison des arrhéphores; spatialement, cette modeste demeure peut être mise en relation avec plusieurs lieux de culte qui, adossés à la falaise septentrionale et orientale de l'abrupt rocher de l'Acropole, jouent un rôle dans la légende héroïque, réélaborée à la fin du $\mathrm{v}^{\mathrm{e}}$ siècle. Tout d'abord il y a la grotte de Pan devant laquelle Euripide situe l'aire de danse au tendre gazon où évoluent les Cécropides; identifiée avec une cavité qui, au pied du rocher, se trouve entre l'aplomb de la maison des arrhéphores et la base septentrionale des Propylées, la grotte est proche de l'endroit où l'on s'imagine que se sont jetées Hersé et Aglauros. S'il est bien correct de l'identifier avec la figure de Courotrophos, Hersé recevrait sans doute des honneurs héroïques dans la grotte située dans la falaise septentrionale de l'Acropole, immédiatement sous la maison des arrhéphores ${ }^{29}$.

28. À partir du premier rapport par Oscar Broneer, «Eros and Aphrodite », sur des fouilles qui ont permis d'identifier le sanctuaire, on verra la synthèse circonstanciée offerte par Vinciane Pirenne-Delforge, L'Aphrodite grecque, p. 48-59; $c f$. encore J. M. Hurwit, The Athenian Acropolis, p. 41-43.

29. Quant à l'hypothétique identification d'Hersé avec Courotrophos, voir le dossier récemment soumis à un nouvel examen par Vinciane Pirenne-Delforge, L'Aphrodite grecque, p. 175-185, à la suite de ses propres considérations de 1994, p. 60-62, et de celles de Pierre Brulé, La Fille d'Athènes, p. 41-43. Le sanctuaire probablement consacré à Hersé a été tour à tour situé dans la maison des arrhéphores 
Quant à Aglauros-Brillance, elle faisait l'objet des honneurs dus à une hérö̈ne dans le sanctuaire où était prêté le serment des éphèbes; on situe désormais ce lieu de culte dans une autre anfractuosité située au nord-est de falaise de l'Acropole, sur ce même flanc escarpé où l'on vénérait aussi, avec Éros, Aphrodite en son jardin, en relation avec l'emplacement de l'agora archaïque située à l'est du rocher sacré. C'est par ce sanctuaire que nous avons commencé notre itinéraire ${ }^{30}$. Au centre géographique de ce dispositif cultuel paysager se dresse, dès le dernier quart $\mathrm{du} \mathrm{v}^{\mathrm{e}}$ siècle, l'Érechthéion où Érechthée luimême est avec Poséidon l'objet d'un culte à la fois héroïque et divin; ce culte est célébré dans le temple même dont son épouse Praxithéa devient la première prêtresse au service d'Athéna Polias, entre olivier et eau de mer surgissant l'un et l'autre du roc aride de l'Acropole ${ }^{31}$. L'action narrative dont des filles de Cécrops sont les protagonistes et l'action cultuelle des petites arrhéphores représentant sans doute les filles des citoyens se déploient donc autour du sanctuaire qui est le symbole aussi bien des valeurs économiques et civiques défendues par Athéna que des valeurs de l'expansion maritime de la cité incarnées par Poséidon, au cœur de l'époque classique ${ }^{32}$.

\section{Une ÉDUCATION ANTHROPOPOIÉTIQUe POUR LeS ATHÉNIENNES}

Essentiels sont donc les échos métaphoriques que se renvoient réciproquement l'espace paysager d'ordre cultuel avec les valeurs

et dans le sanctuaire d'Aphrodite aux Jardins; $c f$. Uta Kron, «Aglauros, Herse, Pandrosos », p. 286.

30. La controverse quant à la situation du sanctuaire dédié à Aglauros fut provoquée par la découverte, plus à l'est de la grotte probablement réservée à Hersé, d'une inscription concernant sa prêtresse : SEG XXXIII (1983), 115; voir le commentaire publié dans SEG XLVI (1996), $137: c f$. Georgios S. Dontas, « The True Aglaurion » et, à sa suite, Greco 1997, p. 623-626, ainsi que J. M. Hurwit, The Athenian Acropolis, p. 204 avec fig. 8; sur Courotrophos, Blauté et Aphrodite, $c f$. Vinciane Pirenne-Delforge, L'Aphrodite grecque, p. 60-62 et 2004, p. $183-184$.

31. Les martúria de l'histoire fondatrice de l'Attique et d'Athènes qui sont vénérés dans le temple d'Athéna Polias dénommé Érechthéion sont mentionnés par Pausanias ( $c f .1,26,5$ et 27, 1-2) à l'occasion de sa visite de l'Acropole; voir déjà Hérodote 8, 55, ainsi que Robert Parker, « Myths of Early Athens », p. 201-204.

32. Sur la collaboration cultuelle et symbolique d'Athéna et de Poséidon en Attique, $c f$. Claude Calame, Thésée, p. 348-355. 
symboliques attribuées aux éléments physiques et végétaux qui le composent, les actes rituels qui s'y déroulent avec les récits héroïques qui les fondent, le statut social de leurs protagonistes, les fonctions et champs d'action des dieux et des héros à qui sont consacrés ces actes de culte. Ces êtres divins sont mis en scène dans les «mythes» étiologiques qui légitiment les pratiques rituelles aussi bien par référence au moment axial et spatio-temporel d'une « première fois » que dans un « ici » où la forme poétique, dans la performance musicale, donne toute son efficacité au récit. De ce point de vue d'une anthropologie historique des pratiques religieuses des Grecs en contexte, il conviendrait, en ce qui concerne l'éducation anthropopoiétique de la jeune Athénienne et les espaces de signification rituelle où elle s'accomplit, d'ajouter au service de l'arrhéphorie en tout cas le double culte rendu à Artémis Braurônia, à Braurôn et sur l'Acropole même, ainsi que les différents cultes dédiés à Déméter et à sa fille entre Athènes et Éleusis.

Paysagers, les sanctuaires correspondants balisent l'espace religieux d'Athènes tout en accueillant et en consacrant, dans et par le rituel, une série de transitions inscrites dans l'itinéraire biologique et social suivi par l'épouse du citoyen : naissance, éducation, puberté, nubilité, union sexuelle, première grossesse, premier accouchement, courotrophie, décès. Marqué par l'éducation chorale qui conduit la jeune fille à l'éclosion de la beauté et au mariage procréateur dans l'accomplissement du désir érotique, ce cursus sollicite, à travers une série d'actes cultuels relevant de la vaste catégorie du rite de passage, des divinités telles qu'Athéna, Artémis, Aphrodite et Déméter avec leurs parèdres souvent hérö̈ques. Dans une série de correspondances assimilant l'effet fertilisant de l'eau claire, la croissance des végétaux et l'adolescence des mortels d'une part, l'effet du travail agricole, la production des aliments de base et la procréation favorisée par la pulsion érotique d'autre part, la femme athénienne est conduite par la constellation des divinités qui la protègent dans son parcours de vie; cet itinéraire va jusqu'à la promesse eschatologique d'une vie se prolongeant dans l'au-delà. Par le biais de l'éducation chorale et par l'intermédiaire des actes rituels se réalise, dans la géographie de l'Attique, un espace transformé en paysage cultuel signifiant par l'activité symbolique et anthropopoiétique des humains.

Laissons à Pausanias le soin de provisoirement conclure : « ̀̀ Grinéion, il y a un très beau bosquet consacré à Apollon : arbres frui- 
tiers et arbres qui n'en donnent pas, plaisir pour l'odorat ou pour le regard »-dit le Périégète d'un Apollinéion qu'il admire en Éolie. Sentiment du paysage sans doute, mais un sentiment religieux dans l'effet esthétique provoqué par un espace qui a été aménagé pour être habité par une divinité du panthéon local, avec ses pouvoirs propres $^{33}$.

Claude.Calame@unil.ch

33. Pausaniasp. 381, 21, 7. 


\section{BIBLIOGRAPHIE}

Francis Affergan, Silvana Borutti, Claude Calame, Ugo Fabietti, Mondher Kilani, Francesco Remotti, Figures de l'humain : Les représentations de l'anthropologie, Paris (Éditions de l'EHESS) 2003.

Claude Bérard, Anodoi. Essai sur l'imagerie des passages chthoniens, Neuchâtel (Institut Suisse de Rome) 1974.

Deborah Boedeker, Descent from Heaven. Images of Dew in Greek Poetry and Religion, Chico (Scholars Press) 1984.

Oscar BRONEER, « Eros and Aphrodite on the North Slope of the Acropolis in Athens », Hesperia 1 (1932), p. 31-55.

Pierre Brulé, La Fille d'Athènes. La religion des filles à Athènes à l'époque classique. Mythes, cultes et société, Besançon - Paris (Annales Littéraires de l'Université de Besançon - Les Belles Lettres) 1987.

Walter BURKERT, « Kekropidensage und Arrhephoria. Vom Initiationsritus zum Panathenäenfest », Hermes 94 (1966), p. 1-25.

Richard Buxton, Imaginary Greece. The contexts of mythology, Cambridge (Cambridge University Press) 1994.

Claude Calame, Thésée et l'imaginaire athénien. Légende et culte en Grèce antique, Lausanne (Payot) 1996 (2 éd.)

Claude Calame, L'Éros dans la Grèce antique, Paris (Belin) 2002 (2éd.).

Claude Calame, Pratiques poétiques de la mémoire. Représentations de l'espace-temps en Grèce ancienne, Paris (La Découverte) 2006.

Claude CALAME, «L'histoire comparée des religions et la construction d'objets différenciés : entre polythéisme gréco-romain et protestantisme allemand ", in M. Burger et C. Calame (edd.), Comparer les comparatismes. Perspectives sur l'histoire et les sciences des religions, Edidit - Archè, Paris - Milano, 2006, p. 209-235.

Claude Calame, «Jardins d'amour et prairies de l'au-delà. Rencontres rituelles avec les dieux et poésie en Grèce antique », Poétique 145 (2006), p. 25-41.

Ada Cohen, « Mythic Landscapes of Greece », in R. D. Woodard (ed.), The Cambridge Companion to Greek Mythology, Cambridge (Cambridge University Press) 2007, p. 305-330. 
Sonia DARTHOU, « Retour à la terre : fin de la geste d'Érechthée », Kernos 18 (2005), p. 69-83.

Marcel Detienne, L'écriture d'Orphée, Paris (Gallimard) 1989.

Ludwig Deubner, Attische Feste, Berlin (H. Keller) 1932.

David B. Dodd \& Christopher A. Faraone (éd.), Initiation in Ancient Greek Rituals and Narratives: New Critical Perspectives, London \& New York (Routledge) 2003.

Guy Donnay, «L'Arrhéphorie : initiation ou rite civique? Un cas d'école », Kernos 10 (1997), p. 177-205.

Georgios S. Dontas, «The True Aglaurion», Hesperia 52 (1983), p. 48-63.

Stella Georgoudi, « Gaia/Gé. Entre mythe, culte et idéologie », in S. des Bouvrie (ed.), Myth and Symbol I. Symbolic phenomena in ancient Greek culture, Bergen (The Norwegian Institute at Athens) 2002, p. 113-134.

Laurent GouRmelen, Kékrops, le Roi-Serpent. Imaginaire athénien, représentations de l'humain et de l'animalité en Grèce ancienne, Paris (Les Belles Lettres) 2004.

Emanuele GRECo, « Definizione dello spazio urbano : architettura e spazio pubblico », in S. Settis (ed.), I Greci. Storia Cultura Arte Società II. Una storia greca 2, Torino (Einaudi) 1997, p. 619-652.

Albert Henrichs, « Dancing in Athens, Dancing on Delos : Some Patterns of Choral Projection in Euripides », Philologus 140 (1996), p. 48-62.

J. M. Hurwit, The Athenian Acropolis : history, mythology, and archaelogy from the noeolithic era to the present, Cambridge (Cambridge University Press) 1999.

Emily Kearns, The Heroes of Attica (Bulletin Supplement 57), London (Institute of Classical Studies) 1989.

Uta Kron, «Aglauros, Herse, Pandrosos », Lexicon Iconographicum Mythologiae Classicae I. 1. Zürich - München (Artemis) 1981, p. 283-298.

Nicole Loraux, Les Enfants d'Athéna. Idées athéniennes sur la citoyenneté et la division des sexes, Paris (La Découverte) 1981.

Nicole Loraux, Né de la terre. Mythe et politique à Athènes, Paris (Seuil) 1996. 
André Moтte, Prairies et Jardins de la Grèce Antique. De la Religion à la Philosophie, Bruxelles (Palais des Académies) 1973.

René NüNLIST, Poetologische Bildersprache in der frühgriechischen Dichtung, Stuttgart - Leipzig (Teubner) 1998.

Mark W. Padilla (ed.), Rites of Passage in Ancient Greece : Literature, Religion, Society, Lewisburg (Bucknell University Press) 1999.

Robert PARKER, « Myths of Early Athens », in J. Bremmer (ed.), Interpretations of Greek Mythology, London - Sydney (Croom Helm Routledge) 1987, p. 187- 214.

Robert PARKer, Polytheism and Society at Athens, Oxford (Oxford University Press) 2005.

Chrysis PÉLÉKIDIS, Histoire de l'éphébie attique. Des origines à 31 avant Jésus-Christ, Paris (de Boccard) 1962.

Franca Perusino, «Le orse di Brauron nella Lysistrata di Aristofane », in B. Gentili \& F. Perusino (edd.), Le orse di Brauron. Un rituale di iniziazione femminile nel santuario di Artemide, Pisa (Edizioni ETS) 2002, p. 167-174.

Vinciane Pirenne-Delforge, L'Aphrodite grecque, Athènes - Liège (CIERGA : Kernos Suppl. 4) 1994.

Vinciane Pirenne-Delforge, « Qui est la Kourotrophos athénienne? », in V. Dasen (ed.), Naissance et petite enfance dans l'Antiquité, Fribourg \& Göttingen (Academic Press - Vandenhoeck \& Ruprecht) 2004, p. $172-185$.

H. Alan Shapiro, « The Daughters of Cecrops », in E. D. Reeder (ed.), Pandora. Women in Classical Greece, Princeton (Princeton University Press) 1996, p. 39-48.

Peter Siewert, « The Ephebic Oath of Fifth-Century Athens », Journal of Hellenic Studies 97 (1977), p. 102-111. 